\title{
Collecting Sensitive Personal Data in a Multi-Cultural Environment
}

\author{
Annika Hinze ${ }^{1}$, Claire Timpany ${ }^{1}$, Judy Bowen ${ }^{1}$, Carole Chang ${ }^{1}$, Nicola Starkey ${ }^{1}$, Hinemoa Elder ${ }^{2}$ \\ ${ }^{1}$ University of Waikato, Hamilton, New Zealand \\ ${ }^{2}$ University of Auckland, Auckland, New Zealand \\ \{hinze,ctimpany,jbowen,starkey,chang\}@waikato.ac.nz, hinemoa@xtra.co.nz
}

\begin{abstract}
Traumatic Brain Injury (TBI) has long-term effects on memory and cognitive functions. This paper discusses the challenges encountered and lessons learned from developing augmented memory aids for people with TBI. In particular, the paper discusses methodologies used to elicit potentially sensitive and personal data.
\end{abstract}

Traumatic Brain Injury, $\mathrm{HCl}$ methodology, qualitative data, participatory design.

\section{INTRODUCTION}

Traumatic Brain Injury (TBI) is one of the most common causes of disability and death in young adults. It has long-term effects on memory and other cognitive functions (Feigin, 2010). Memory impairment and other long-lasting cognitive difficulties are one of the most common symptoms of TBI and affect many TBI survivors (Barker-Collo, 2008). Longterm care and support for people with TBI is complex and affects not only the person with the injury but also their close family and friends, and the community. Memory problems and impaired cognition often last well beyond the initial period of recovery and are cited by patients and caregivers as one of the greatest sources of issues in daily life. They may often add more to lasting disability than physical issues even years after the injury; TBI is thus the beginning of a chronic disease process (McCullagh, 2011). This paper is a collaboration between researchers in computer science, cognitive psychology and health care, in which we describe our experience with developing autobiographical memory aid systems for people with TBI. In particular, we focus on the design process we followed to elicit requirements and feedback throughout the development. We describe the challenges encountered and the methods we used to overcome these.

Here we briefly list the potential challenges when designing autobiographical memory aids.

- Naturally when testing these aids, records of the participants' personal daily lives are recorded.

- Exploring issues and memory problems exposes and highlights participants' limitations

- Cultural expectations may vary about acceptable personal questions for experiences with $\mathrm{TBI}$, and how to understand health and impairment

- Participants may feel pressured to justify their healing progress and ability to manage
- Many caregivers are family members, so issues of privacy and personal life may be mingled

- Some participants may wish not acknowledge or are unaware of their memory limitations

Section 2 describes the two memory aids we developed, while Section 3 outlines our study methods and observations. Section 4 discusses challenges of our research in culturally-diverse contexts. Section 5 , summarises our lessons learned, and the paper ends with a short conclusion regarding participatory design with and for people with TBI.

\section{DIGITAL AUGMENTED MEMORY AIDS}

Our research aims to develop augmented memory aids and therapeutic games that may support people with $\mathrm{TBI}$ and their families. We have developed the mobile augmented memory app MyMemory, which aims to improve TBI survivors' memory and support them in their daily lives (Chang, 2017). MyMemory helps people record memory cues about events in their lives, which may be looked up later to trigger remembering. It also offers memory training based on these memory cues, using the concept of memory training flashcards and memory-ticker widgets. MyMemory was designed using qualitative methods and human-centred design approaches in collaboration with TBI survivors. We further explored a community-focussed memory system in which both TBI survivor and their families/caregivers encode memory cues (Khong, 2016). This may help survivors to extend the range of memories to which they have access. Here a group of people can contribute memory cues that relate to earlier life spans of the TBI survivor, who may or may not remember the event themselves. 


\section{DESIGN METHODS USED}

Throughout the course of our research, we used several user-centred design methods, which we briefly describe here. We will reflect on impressions from both researchers as well as study participants. Our work did not include access to medical records.

Study in online community: In order to understand how symptoms affect the daily lives of people with $\mathrm{TBI}$, we performed a survey on the online platform 'PartientsLikeMe.com'. We registered with the website as researchers, which gave structured access to self-reported medical data for statistical purposes. 282 of over 100,000 registered patients report suffering from TBI, with 207 of these reporting memory problems. The collected data also confirmed that brain injury does not occur in isolation but often leads to other conditions including epilepsy, migraine, major depressive disorder, and generalized anxiety disorder (GAD), fibromyalgia and exhaustion. We further found that patients reported that their brain injury caused further symptoms such as difficulty concentrating, vision problems, fatigue and cognitive disorders, many of which lead to participants having to significantly change their daily lives.

Interview Study: We performed an interview study to elicit information about (1) the special requirements TBI survivors have for the design of an augmented memory aid, (2) the TBI survivors' existing memory aids and strategies. We used both face to face interviews (for participants within $1 \mathrm{~h}$ drive) and online questionnaires (for participants who lived further afield). We aimed to include both TBI survivors and caregiver participants. We used open recruitment of participants via posters and TBI mailing lists. Recruitment presented a particular challenge because the NZ Privacy Act 1993 states that organisations (such as doctors, hospitals and TBI organisations) are not permitted to provide details of any individuals. Furthermore, recruitment within hospital areas was not permitted without the very elaborate specific ethical approval process geared towards clinical studies. Due to the vulnerability and, in some cases, isolation and loneliness of people with TBI, the overall recruitment process was severely hampered. The recruitment and interviewing of 34 participants took 4 years. The study allowed us to learn about specific problems encountered (remembering new acquaintances, ad-hoc events, and instructions) and memory strategies used. The strategies were often personalised and geared towards providing support in specific settings, such as reliance on partners for memory cues and reminders, physical notes (on paper or on the body) and digital notes. Desktop-based systems were not suitable (often causing head-aches) and established systems often too complex. We learned that the three most important characteristics for a new memory aid would therefore be customisability, simplicity, and discreetness. The TBI participants were very concerned not to disclose their condition involuntarily and therefore avoid feeling embarrassed.

Personas, scenarios, and participatory prototype design: Based on the insights from our study and interviews, we developed personas (very closely mirroring some of the TBI participants) and usage scenarios for both single-use (i.e., TBI survivor only) and community use (i.e., joint contribution to memory cues). The aim was to not overburden the participants who expressed their willingness to be closely involved in the design process. We were in regular contact with them to probe the suitability and realism of the scenarios we used for the design process. Using the scenarios, we identified more detailed technical requirements. For example, the interface and interaction design needed to be very clear and not contain intricate (but ultimately merely illustrative / decorative) details. Based on design sketches (paper prototypes) we developed digital paper prototypes using the tool Balsamiq, which supports interactive wireframe models. These simple interface models were tested in a user study with participants with and without TBI. The reason was that we did not wish to overburden possible TBI participants with too many design feedback tasks. Again the recruitment process was lengthy and challenging. As the study had to be executed at the University (for technical reasons), the participants needed to rearrange their schedules and some of their health regimes to allow for travel to the study. Some participants even travelled to the university during the previous week to train themselves into finding the way (so that they would be on time for the user study meeting). Health issues that some TBI-participants reported included low energy and functioning concentration only at certain times (e.g. before $11 \mathrm{am}$ ), or having a minor illness but needing a recovery time of one week or more. Therefore, successfully arranging a meeting included on average at least three changes, each change needed up to three days of waiting for a reply to email. Overall the recruitment and study with 9 participants took eight months to organise.

Based on our experiences with this first prototype study, for our second, community-based application, this phase was conducted as a small focus group involving two participants (one TBI survivor and their caregiver). The advantage was closer participation and collaboration over some time but less variation in the feedback received. We initially explored alternative design layout variations, which was then followed by in-depth design discussions and use case explorations. These were somewhat guided by the researchers, but largely led by the participants. This process led to a number of design suggestions regarding interface, interaction and conceptual support (e.g., identifying contributors of memory cues, adding a place for peer-to-peer reflection within the community, linkages between memories, and randomised memory of the day). This worked particular- 
ly well for the relatively open design of the community based app (capture a variety of memory cues that may be helpful for the TBI survivor and their family). For the first systems (single user), the design was more purposeful (as simple as possible to quickly and unobtrusively capture necessary memory cues during the day), and aimed to avoid complexity and identify the essentials.

Digital Prototype usage exploration: The community-based app was implemented based on improved wireframe models and focus group feedback. The app was brought back to the focus group to explore and use over a three week time period, followed by an interview. The participants added a number of memory cues together throughout the study. However, the time period was found to be too short to collaboratively develop a useful collection of memory cues. We had previously observed this problem of evaluating systems that rely on the participants forgetting or targeting longer time frames for memories (i.e., memories about childhood not about yesterday's events) in our research into autobiographical memory aids (Schweer, 2009). Any study first needs to collect a sufficient number of memories (not merely data that is looked up) followed by sufficient time for participants to forget. Obtaining such personal data and participants willing to engage in a very personal process over a potentially very long time is challenging to organise.

Overall, the feedback about the community-based interaction and interface design was positive (appreciation for the minimalistic design, ease of use while travelling). Both focus group and user study strongly emphasised the need for customisation (alternative sequences of interactions), which was not possible to provide under the circumstances.

Prototype in use (ABAB study): The MyMemory digital prototype was tested in an ABAB-type study (Ferguson, 2015) in which phases of intervention (using MyMemory) were interleaved with baseline phases in which participants returned to their own memory aid strategies. Each phase took 2 weeks (overall 8 weeks) for each TBI participant. The study had six TBI participants and 3 caregiver participants; we evaluated the perceived memory ability of the TBI participants and the well-being of all participants. Analysis of memory records and log reports showed that most TBI participants provided full details of each memory in MyMemory. All of them used photos in most of their memory records, which they reported to provide important cues. Overall, $532 \mathrm{mem}$ ories were captured during the intervention phases. The study design meant that the TBI participants changed their memory aids every fortnight. This is particularly challenging for TBI survivors who rely on consistency in their approaches to support their memory, taking part in the study therefore had the potential to increase the amount of stress in their daily life. This switching between memory aids may have an effect on the results and the feelings of participants towards using the app. A better solution for future studies may be to investigate the use of longer, single-person studies, to try and measure the effects of using the app consistently over longer periods of time.

\section{CULTURAL AWARENESS}

The two approaches described so far used established methods from $\mathrm{HCl}$ and psychology. In none of these did we address any questions of cultural awareness. In New Zealand, Māori people are disproportionately affected by TBI, with Māori men being at four times the risk of the general population, and they also face the risk of poorer outcomes (Barker-Collo, 2008). Studies have shown that different cultural practices, practitioner insensitivity towards cultural practice, socioeconomic status, communication issues and patients' personal experiences were contributing factors to poor treatment outcomes (Davis, 2006). Given these known challenges in established health care practices, how should software design be approached when dealing with such sensitive and personal data as autobiographical memories? Māori traditionally hold a more holistic view towards health than Caucasians. Māori prefer to seek treatment within their whanau (extended family) instead of seeking medical help in the hospital (Elder, 2013).Thus a community-based approach to design may also be more suitable. One needs to take guidance from the Māori culture, and by doing so, we may be provided a better understanding of a participant's background and suitable approaches to participatory design.

From a Māori world view perspective the importance of the head cannot be overemphasised. The wellknown belief "he tapu te upoko" (the head is sacred) continues to be recognised by most Māori according to recent research (Elder, 2013). Addressing the cultural impact of TBI has been the recent focus of one of the author's research and two tools have been developed, Te Waka Kuaka and Te Waka Oranga, which are now in use in rehabilitation facilities (Elder, 2017)). In addition, the importance of time taken in assessment has also recently been reported (Elder, 2016). This has significant implications for the development of tools which recognise Māori cultural practices to ensure the success of assessment and intervention practices.

\section{INSIGHTS AND CONCLUSIONS}

To create a trusting environment and build a rapport with the participants, as well as overcoming memory challenges, it is beneficial to use the participants' familiar location instead of a research laboratory. In a multi-cultural environment, increased care is required in developing relationships - within the confines of the work so far we have not explored these in depth. Ideally one of the researchers is part of the 
culture and can provide means of instruction that are suitable. Both $\mathrm{TBI}$ participants and caregivers were asked to describe memory impairments and issues encountered. It did not appear as if any participants wished to hide any issues but sometimes they were not aware of their limitations (which were brought up by a caregiver). For all participants it is a normalised experience to encounter memories reported by other people. However, caregiver and patient relationships differ heavily depending on the social dynamics (e.g. the son who cares for his mother). A caregiver commented there some issues she would raise in a one-on-one interview that may have been embarrassing in a joint interview. Conversely, her partner commented "when we were interviewed together I was sometimes surprised by my partner's answers." Many TBI participants had already had to accept repeated loss of privacy to caregivers and health professionals. The participants seemed to have moved beyond worrying about embarrassment. Our team never asked participants about adherence to clinical rehabilitation work. It was a prerequisite for participation that main medical issues had been resolved.

Participants volunteered for our studies; they were willing to share information with the team. The type and amount of data participants entered into the apps was their own choice. We are naturally not aware of information that is left out. However, no participant ever expressed any privacy concerns or mentioned the invasive nature of our procedures. When the participants start recording their memory cues, we had already done several interviews with them, most of which happened in their own home. To some extent, we had become part of their support network and integrated into their care routine. Participants who may have had concerns could have dropped out of the studies, however we could not directly track this as participants frequently encountered a number of health and organisational issues. Overall, it appeared that the opportunity to gain access to a tool that may help with their memory was a stronger driver than potential fears of exposing private memories or limitations.

Initially it seemed to be a challenge not to have access to medical records, refined tools for measuring memory improvements and large numbers of participants. However, we found this actually led to better relationships in which both the researchers and the participants had the opportunity to engage more fully. We therefore never aimed to objectively measure improved memory but noted the participants' selfreported changes in memory and well-being. Due to the time and intensity of interactions with the participants, it seems more likely that the responses were honest. We believe that observing and engaging with a smaller group of people over a longer period of time leads to better qualitative outcomes. However, it is draining and difficult for people with TBI to intensely engage with the process for a long time, so building a small community of participants could be a viable alternative. Furthermore, as each person's situation is so different, adding more people may not necessarily be a viable option. It is the hope that when designing software for, and with, a small group of people with in-depth understanding, the resulting software may work similarly for others.

The collaboration with colleagues in cognitive psychology was immensely beneficial for our projects. However, the expectations outside of user-centred design may often call for large numbers of participants and quantitative exploration whereas participatory design is largely qualitative.

Finally, for memory apps to be used long-term, they most likely need to offer some degree of privacy, e.g., a way to remember private events or thoughts. They could become like a diary or confidante. This may require user groups and access right management to provide layers of access.

Acknowledgements. We wish to thank the study participants, and acknowledge Emily Kong, who implemented and tested the community-based app.

\section{REFERENCES}

Barker-Collo, S., Wilde, N., Feigin, V., (2008) Trends in head injury incidence in New Zealand: A hospital-based study from $1997 / 1998$ to 2003/2004. Neuroepidemiology 32 (1), 32-39.

Chang, C. S.-P., (2017) A mobile augmented memory aid for people with traumatic brain injury. Ph.D. thesis, University of Waikato, Hamilton, New Zealand

Davis, P., Lay-Yee, R., Dyall, L., et al. (2006). Quality of hospital care for Māori patients in New Zealand: retrospective crosssectional assessment. The Lancet, 367(9526), 1920-1925.

Elder, H. (2013). Indigenous theory building for Māori children and adolescents with traumatic brain injury and their extended family. Brain Impairment, 14(03), 406-414.

Elder, H., Kersten, P, McPherson, K., et al. (2016) Making time, deeper connection, fuller stories, best practice. Experiences of Māori and non-Māori nurse researchers. Annals of Psychiatry and Mental Health 4(6), 1079-1082.

Elder H. (2017) Te Waka Kuaka and Te Waka Oranga. Working with whānau (extended families) to improve outcomes. Australia and NZ Journal of Family Therapy, 38:1, 27-42

Feigin, V., Barker-Collo, S., Krishnamurthi, R., Theadom, A., Starkey, N., (2010) Epidemiology of ischaemic stroke and traumatic brain injury. Best Practice and Research Clinical Anaesthesiology 24 (4), 485-494.

Ferguson, S., Friedland, D., Woodberry, E. (2015). Smartphone technology: Gentle reminders of everyday tasks for those with prospective memory difficulties post-brain injury. Brain Injury, 29(5), 1-9.

Khong, E., (2016) Exploring community-based health care using mobile applications. Master's thesis, University of Waikato.

McCullagh, S., Feinstein, A., (2011) Cognitive changes. Vol. 2. American Psychiatric Press, pp. 279-294.

Schweer, A. (2011). Augmenting Autobiographical Memory: An Approach Based on Cognitive Psychology. Ph.D. thesis, University of Waikato, Hamilton, New Zealand. 